\title{
Oral Health Related Quality of Life Among Adult Patients Attending Periodontal Clinic in Kuliyyah of Dentistry IIUM Kuantan
}

\author{
Juzaily binti Husain, Farah Natasha Mohd, Abdul Hadi Said, Munirah Yaacob \\ International Islamic University Malaysia
}

Introduction: There was lack of local study assessing the oral health related quality of life (OHRQOL) of patients with periodontal diseases. Hence, this study aims to assess the OHRQOL and its associated factors in adult patients who were diagnosed with gingivitis and periodontitis. Material and method: This was a cross sectional study conducted from May to December 2018 among 100 participants attended Periodontal Clinic, Kulliyyah of Dentistry of International Islamic University of Malaysia (IIUM) using purposive sampling methods. Clinical parameters of participants were recorded by a single examiner then followed by a selfadministered questionnaire using the validated short version of Oral Health Impact Profile(S-OHIP) (Malay) to assess the OHRQOL and its associated factors. The OHRQOL of participants were reported using mean and standard deviation. The factors associated with OHRQOL were determined using independent t-test and Spearman correlation test. Results: The mean score for S-OHIP was 14.73 \pm 9.24. Participants with periodontitis have higher S-OHIP score compared to those with gingivitis. There were significant association between OHRQOL and underlying diabetes mellitus, self-perceived oral health status and self-perceived oral health satisfaction with $p$ value $<0.05$. This shows that those with underlying diabetes mellitus have higher S-OHIP score indicated poorer OHRQOL. Meanwhile, those who perceived they have either good or very good oral hygiene and satisfied with their oral health have lower S-OHIP score indicated better OHRQOL. Conclusion: This study found that periodontal diseases have significant association with the OHRQOL. Therefore, reinforcement of oral health education by the dentists is crucial. 